\title{
Crystal structure, magnetic and thermal properties of $\mathrm{UCoSnD}_{\mathbf{0 . 6}}$
}

\author{
Khrystyna MILIYANCHUK ${ }^{1,2 *}$, Ladislav HAVELA ${ }^{2}$, João C. WAERENBORGH ${ }^{3}$, Piotr M. GACZYŃSKI ${ }^{3}$, \\ Oleksandr PROKHNENKO ${ }^{4}$ \\ ${ }^{1}$ Department of Inorganic Chemistry, Ivan Franko National University of Lviv, \\ Kyryla i Mefodiya St. 6, 79005 Lviv, Ukraine \\ ${ }^{2}$ Department of Condensed Matter Physics, Faculty of Mathematics and Physics, Charles University, \\ Ke Karlovu 5, 12116 Prague 2, Czech Republic \\ ${ }^{3}$ Departamento de Química, Instituto Tecnológico e Nuclear/CFMC-UL, P-2686-953, Sacavém, Portugal \\ ${ }^{4}$ Hahn-Meitner Institute, SF-2, Glienicker Str. 100, 14109 Berlin, Germany \\ *Corresponding author.Tel.: +380-32-2394506; e-mail: k_miliyanchuk@franko.lviv.ua
}

Received November 12, 2007; accepted May 20, 2008; available on-line September 10, 2008

The new deuteride $\mathrm{UCoSnD}_{0.6}$ was investigated by neutron powder diffraction, magnetic measurements, specific heat studies, and Mössbauer spectroscopy. It was shown that the insertion of deuterium atom inside the $\mathrm{U}_{3} \mathrm{Co}$ tetrahedra with concomitant lattice expansion by $3.2 \%$ leads to a pronounced enhancement of magnetism. The reason for such a behavior is a partial localization of the electronic states, which is reflected in the increase of the density of states at the Fermi level and increase of the $s$-electron density on the Sn nuclei.

Deuteride / Uranium / Neutron diffraction / Specific heat / Mössbauer spectra

\section{Introduction}

UCoSn (ZrNiAl structure type, $a=7.148 \AA$, $\left.\mathrm{c}=3.99 \AA \quad[1], \quad T_{\mathrm{C}}=80-88 \mathrm{~K} \quad[2-5]\right)$ was already reported to absorb hydrogen [6]. The formed hydride $\mathrm{UCoSnH}_{1.4}$ is characterized by a pronounced enhancement of the magnetism. The Curie temperature increases from $82 \mathrm{~K}$ to $102 \mathrm{~K}$ and the spontaneous magnetic moment in randomly oriented powder sample increases from $0.64 \mu_{\mathrm{B}} /$ f.u. to $0.75 \mu_{\mathrm{B}} /$ f.u. However, studies of the isostructural URuSn compound surprisingly revealed an opposite effect. URuSnH$H_{1.4}$ exhibits a decrease of both the Curie temperature, from $54 \mathrm{~K}$ to $51 \mathrm{~K}$, and the spontaneous magnetic moment, from $0.53 \mu_{\mathrm{B}} /$ f.u. to $0.48 \mu_{\mathrm{B}} / \mathrm{f}$.u [6]. To explain the different behavior of these compounds upon hydrogenation we suggested that hydrogen atoms occupy different positions. In the present work we summarize our results from the study of the $\mathrm{UCoSnD}_{0.6}$ deuteride. The prime task was to determine the deuterium positions by a neutron powder diffraction experiment. Besides, the study of such properties as specific heat and Mössbauer spectra provided additional details on the modification of the electronic structure caused by deuteration.

\section{Experimental details}

The synthesis of $\mathrm{UCoSnD}_{0.6}$ was preceded by the preparation of corresponding intermetallic compound. The sample was prepared by arc-melting the stoichiometric amount of metals of purity at least 99.9\% under argon atmosphere. The deuteride was synthesized by exposing an activated UCoSn sample to deuterium gas at $p=120 \mathrm{bar}$ and subsequent thermal treatment up to $T=923 \mathrm{~K}$. To quantify the amount of absorbed deuterium, a small amount of deuteride was decomposed in vacuum in a closed volume by heating up to $1023 \mathrm{~K}$. The phase composition of the initial and the deuteride samples was checked by X-ray powder diffraction (XRD-3003 Seifert diffractometer, $\mathrm{Cu}-K \alpha$ radiation, step $0.02^{\circ}$ of $2 \theta)$.

The crystal structure of $\mathrm{UCoSnD}_{0.6}$ was studied by neutron powder diffraction. The data were collected at Berlin Neutron Scattering Center, Hahn-MeitnerInstitute (fine resolution E9 diffractometer, focusing composite germanium monochromator with reflecting plane (511), $\lambda=1.797 \AA$, step $0.08^{\circ}$ of $2 \theta$ ). The measurements were performed at room temperature and the task was to determine the deuterium positions. 
Table 1 Lattice parameters $a$ and $c$, and unit cell volume $V$ of $\mathrm{UCoSn}, \mathrm{UCoSnH}_{1.4}$ (both X-ray data), and $\mathrm{UCoSnD}_{0.6}$ (neutron diffraction data) at room temperature, atom coordinates, displacement parameters $B$ and coefficients of the site occupancy $n$ of $\mathrm{UCoSnD}_{0.6}\left(R_{\mathrm{Bragg}}=6.22 \%, R_{\mathrm{p}}=4.50 \%\right)$.

\begin{tabular}{|c|c|c|c|c|c|c|}
\hline \multicolumn{2}{|l|}{$\begin{array}{l}\mathrm{UCoSn} \\
\mathrm{UCoSnH}_{1.4} \\
\mathrm{UCOSnD}_{0.6}\end{array}$} & $\begin{array}{l}a=7.144(1) \AA \\
a=7.2386(6) \AA \\
\Delta a / a=1.3 \% \\
a=7.2332(2) \AA \\
\Delta a / a=1.2 \%\end{array}$ & \multicolumn{2}{|c|}{$\begin{array}{l}c=3.993(1) \AA \\
c=4.0189(5) \AA \\
\Delta c / c=0.7 \% \\
c=4.0208(2) \AA \\
\Delta c / c=0.7 \%\end{array}$} & \multicolumn{2}{|c|}{$\begin{array}{l}V=176.5 \AA^{3} \\
V=182.4 \AA^{3} \\
\Delta V / V=3.3 \% \\
V=182.2 \AA^{3} \\
\Delta V / V=3.2 \%\end{array}$} \\
\hline Atom & Site & $x$ & $y$ & $z$ & $B\left(\AA^{2}\right)$ & $n$ \\
\hline $\mathrm{U}$ & $3 f$ & $0.5993(8)$ & 0 & 0 & 0.34 & 1 \\
\hline Co1 & $1 a$ & 0 & 0 & 0 & 0.56 & 1 \\
\hline $\mathrm{Co} 2$ & $2 d$ & 0.3333 & 0.6667 & 0.5 & 0.56 & 1 \\
\hline $\mathrm{Sn}$ & $3 g$ & $0.2581(9)$ & 0 & 0 & 0.40 & 1 \\
\hline $\mathrm{D}$ & $4 h$ & 0.3333 & 0.6667 & $0.0634(39)$ & 1.13 & $0.456(6)$ \\
\hline
\end{tabular}

The evaluation of both X-ray and neutron diffraction data was based on the Rietveld algorithm using FullProf software [7].

Measurements of DC magnetization were performed on randomly oriented fixed powder in the temperature range 5-300 $\mathrm{K}$ and applied external fields up to $4 \mathrm{~T}$ by means of a Quantum Design SQUID magnetometer.

The heat capacity measurements were performed using the Quantum Design PPMS in the temperature range $1.8-300 \mathrm{~K}$. The measurements were performed on pellets prepared from deuteride powder by pressing in a hydraulic press. A special anvil cell with WC faces made it possible to reach several hundreds MPa, which turned out to be sufficient to produce mechanically stable thin pellets.

Mössbauer spectroscopy was performed at the Instituto Tecnológico e Nuclear, Sacavém, Portugal. Mössbauer spectra were collected in transmission mode using a conventional constant-acceleration spectrometer and a $10 \mathrm{mCi}{ }^{119 m} \mathrm{Sn}$ source in $\mathrm{CaSnO}_{3}$; the velocity scale was calibrated using a ${ }^{57} \mathrm{Co}$ source and an $\alpha-F e$ foil. The absorbers were obtained by pressing the powdered samples into perspex holders. The absorber thickness was calculated on the basis of the corresponding electronic mass-absorption coefficients for $23.9 \mathrm{keV}$ radiation. For the data presented in this work, the isomer shifts $(I S)$ are given relative to the $\mathrm{CaSnO}_{3}$ source. Low-temperature spectra were collected using a liquid-helium flow cryostat with a temperature stability of $\pm 0.5 \mathrm{~K}$. The spectra were fitted to Lorentzian profiles using a nonlinear least-squares method [8]. The positions and relative intensities of the absorption lines of the $\mathrm{Sn}$ atoms with non-zero transferred magnetic hyperfine field were calculated by solving the complete Hamiltonian for the hyperfine interactions in the excited and ground nuclear states of the ${ }^{119} \mathrm{Sn}$ nuclei, following the procedure described by Ruebenbauer and Birchall [9].

\section{Results and discussion}

$X$-ray and neutron diffraction

The X-ray powder diffraction patterns of the initial alloy showed the presence of UCoSn as the major phase and a certain amount $(\approx 11 \%)$ of a spurious phase, $\mathrm{UCo}_{\sim 1.4} \mathrm{Sn}_{\sim 1.85}$ [10] $\left(\mathrm{CaBe}_{2} \mathrm{Ge}_{2}\right.$ structure type, space group $P 4 / \mathrm{nmm}, a=4.387 \AA, c=9.040 \AA$ ). The spurious phase remains unaffected by the deuteration, as was seen from the comparison of the diffraction patterns of the samples before and after the deuteration.

An interesting fact was revealed by the decomposition study of the UCoSn deuteride. The decomposition in vacuum by heating up to $1023 \mathrm{~K}$ yielded a stoichiometry of $0.7(1) \mathrm{D}$ atoms per formula unit (versus $1.4 \mathrm{H}$ atoms/f.u. for the corresponding hydride), while the X-ray diffraction revealed that the lattice parameters of the deuteride and the hydride are very close to each other (Table 1). In order to explain this peculiar effect we assume tentatively that a less stable hydride with twice as much hydrogen is formed initially, but decomposes rapidly into a lower hydride. This process may be assisted by the mechanical treatment (crushing) before the $\mathrm{X}$-ray diffraction pattern is collected. The X-ray analysis of the decomposed sample proved that the full desorption state is indeed achieved for the deuteride. Furthermore, the refinement of the neutron powder diffraction data mentioned below showed that the $\mathrm{UCoSnD}_{0.6}$ stoichiometry is correct.

The neutron diffraction pattern of $\mathrm{UCoSnD}_{0.6}$ taken at room temperature is shown in Fig. 1. The crystal structure refinement showed that the metal atoms form a ZrNiAl-type structure (space group $P \overline{6} 2 m$ ), similar to that of the initial compound but expanded by $3.2 \%$. The crystal structure parameters are given in Table 1. The lattice expansion is anisotropic, with prevailing basal-plane expansion. The deuterium atoms occupy the $4 h$ position inside the 


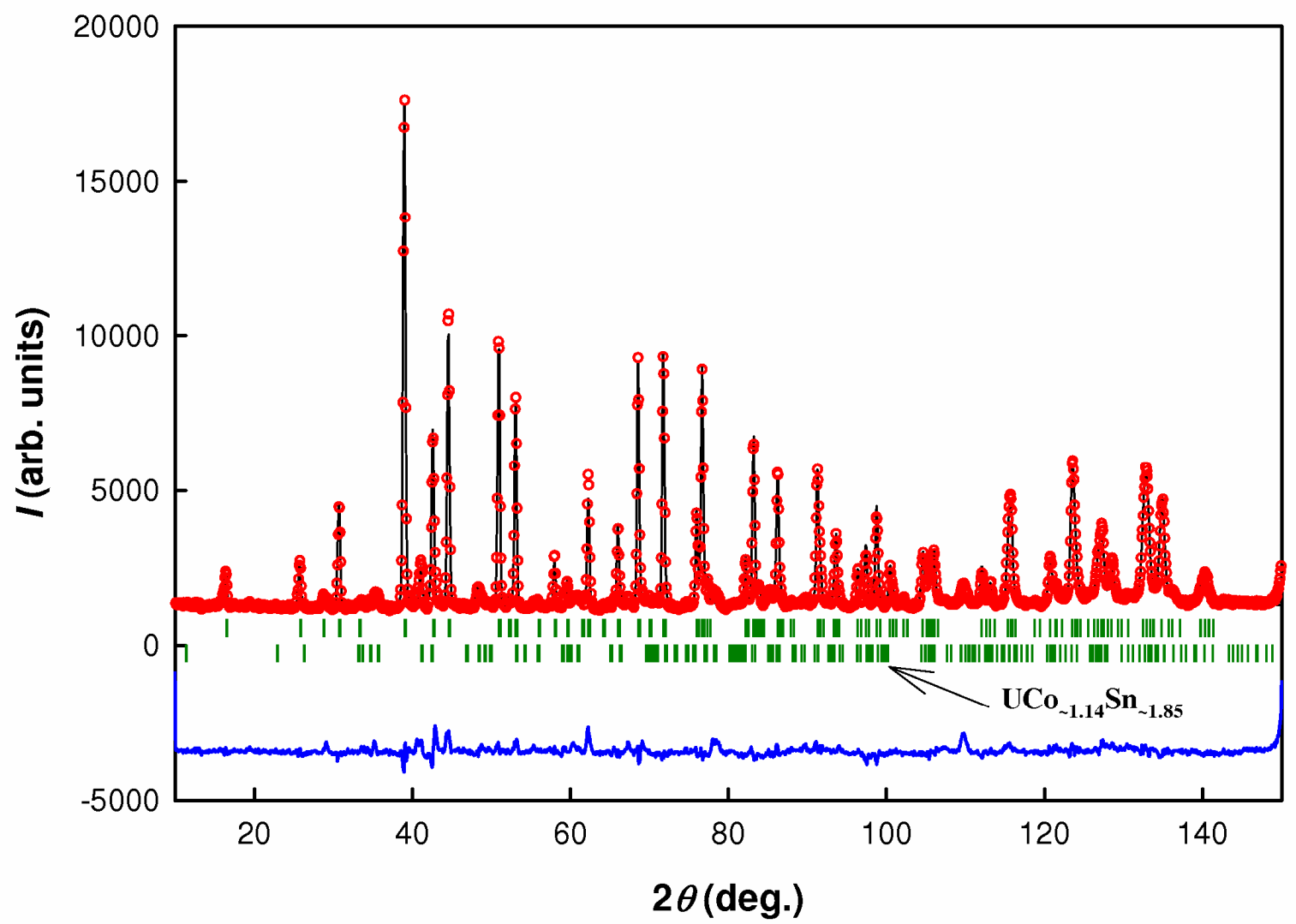

Fig. 1 Neutron diffraction pattern of $\mathrm{UCoSnD}_{0.6}(\lambda=1.797 \AA)$. The observed values (circles), the calculated values (solid line), difference pattern and the positions of the Bragg reflections of $\mathrm{UCoSnD}_{0.6}$ and the impurity phase are shown.

$\mathrm{U}_{3} \mathrm{Co}$ tetrahedra. The crystallographic stoichiometry was refined as $\mathrm{UCoSnD}_{0.608(8)}$, leading to a smaller deuterium content as compared with the stoichiometry determined by the volumetric method (0.7(1) D atoms/f.u.), but both values are comparable within the experimental uncertainty. This structure was already found for $\mathrm{UNiAlD}_{0.7}$ [11] - a non-saturated deuteride of the isostructural compound UNiAl. The crystal structure of $\mathrm{UCoSnD}_{0.6}$ is presented in Fig. 2. The $\mathrm{U}_{3} \mathrm{Co}$ tetrahedra, which accommodate the deuterium atom, share a face, and according to "Shoemaker's exclusion rule" [12] both of them cannot be occupied at the same time. Besides, the distance between two deuterium positions $(0.51 \AA)$ does not allow simultaneous occupancy of neighboring sites [13]. No additional reflections corresponding to possible ordering of the deuterium atoms were observed, therefore we suggest that the deuterium atoms occupy one out of two neighboring interstitial sites randomly. However, this model seems to fail for the mentioned hydride with the stoichiometry $1.4 \mathrm{H} / \mathrm{f}$.u., leading to extremely short distances between the hydrogen atoms, what might be the reason of its instability. In order to satisfy the rule of minimum hydrogenhydrogen distances $2.1 \AA$, the hydrogen atoms should be shifted apart $\left(z_{\mathrm{H}}>0.2\right)$.
Deuterium atoms located inside the tetrahedra consisting of uranium and cobalt occupy a very typical position for metal hydrides. It is known that the crucial parameter that predetermines the magnetic properties of uranium intermetallides is the degree of $f$ - $d$ hybridization. The intrusion of hydrogen atoms, leading to an expansion of the unit cell and to modifications of the electronic structure via the formation of new bonds with its nearest neighbors, must have straightforward impact on magnetic properties of UCoSn.

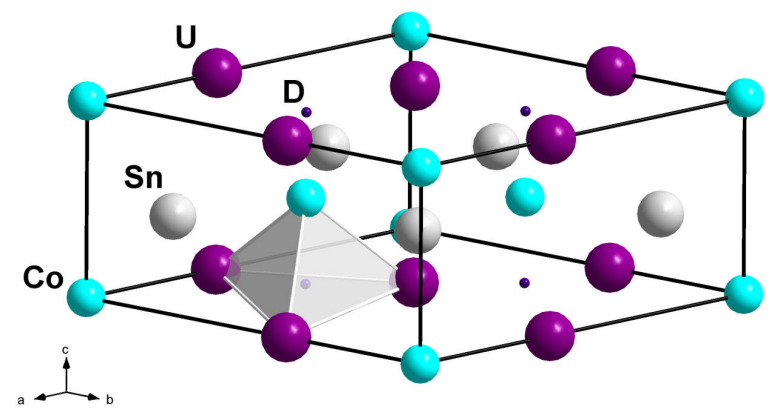

Fig. 2 Crystal structure of $\mathrm{UCoSnD}_{0.6}$. 


\section{Magnetic properties}

The measurements of the magnetic susceptibility showed that the magnetic properties of $\mathrm{UCoSnD}_{0.6}$ are very close to those of the corresponding hydride [6]. $\mathrm{UCoSnD}_{0.6}$ orders ferromagnetically, similarly to the hydride and the initial compound. In the paramagnetic range, the susceptibility curve is described by a modified Curie-Weiss law (Fig. 3). The fit parameters are presented in Table 2. The paramagnetic Curie temperature is slightly higher than that of the hydride $\left(\theta_{\mathrm{P}}=97 \mathrm{~K}\right.$ for the hydride) and the same is true for the Curie temperature determined from the detailed lowfield magnetic susceptibility measurements. The increase of the Curie temperature by approximately $2 \mathrm{~K}$ may be attributed to a small, but non-zero homogeneity region due to the non-stoichiometric composition of both the hydride and the deuteride.

Taking into account the reproducibility of the magnetic properties we may conclude that all the structure data obtained for the deuteride should also be valid for the hydride. However, it does not solve the puzzle with the apparent difference between the stoichiometry of the hydride and the deuteride.

Table 2 Curie temperature $T_{\mathrm{C}}$ and parameters of the modified Curie-Weiss fit of the hightemperature susceptibility (effective moments $\mu_{\text {eff }}$, paramagnetic Curie temperature $\theta_{\mathrm{P}}$, $T$-independent term $\chi_{0}$ ) for UCoSn and the corresponding deuteride.

\begin{tabular}{l|c|c}
\hline & $\mathrm{UCoSn}$ & $\mathrm{UCoSnD}_{0.6}$ \\
\hline$T_{\mathrm{C}}(\mathrm{K})$ & 82 & 104 \\
$\mu_{\text {eff }}\left(\mu_{\mathrm{B}} /\right.$ f.u. $)$ & 2.0 & 2.1 \\
$\theta_{\mathrm{P}}(\mathrm{K})$ & 77 & 102 \\
$\chi_{0}\left(10^{-8} \mathrm{~m}^{3} / \mathrm{mol}\right)$ & 1.3 & 1.1 \\
\hline
\end{tabular}

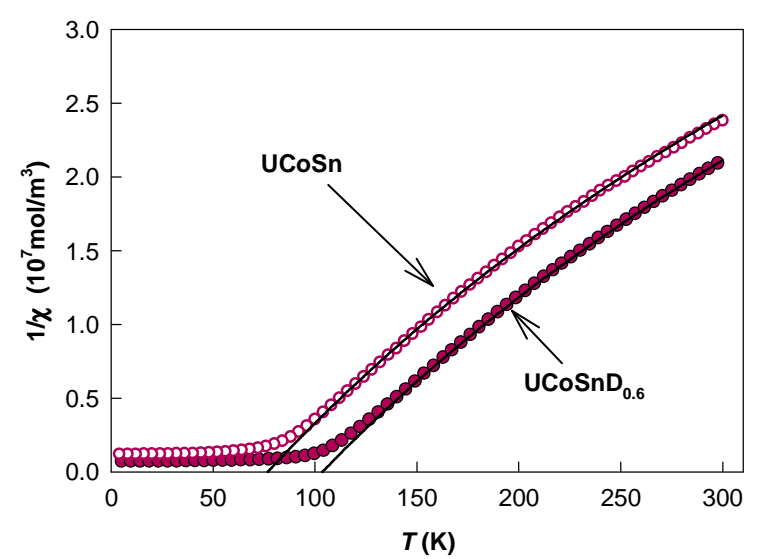

Fig. 3 Temperature dependence of the inverse magnetic susceptibility of UCoSn (measured in magnetic field $6 \mathrm{~T}$ ) and of $\mathrm{UCoSnD}_{0.6}$ (measured in magnetic field $4 \mathrm{~T}$ ). The solid lines represent the modified Curie-Weiss fit (see Table 2 for the corresponding parameters).

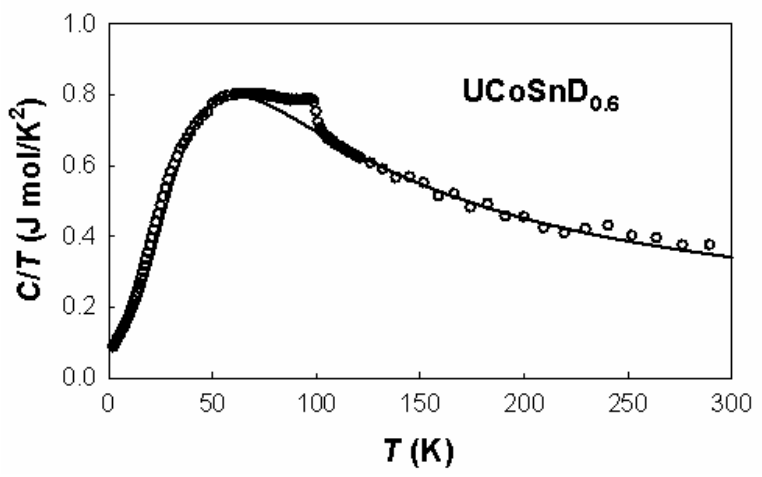

Fig. 4 Temperature dependence of the specific heat of $\mathrm{UCoSnD}_{0.6}$. The solid line represents total fitted electron and phonon contributions.

\section{Specific heat measurements}

Specific heat data were collected for $\mathrm{UCoSnD}_{0.6}$ down to $T=2.2 \mathrm{~K}$ (Fig. 4). The temperature dependence is characterized by a pronounced anomaly at the temperature of the magnetic phase transition.

The $\gamma$-coefficient of electronic specific heat was determined by linear extrapolation of $C_{\mathrm{P}} / T v s . T^{2}$ to $T=0 \mathrm{~K}$. The value of $\gamma$ increases upon deuteration and reaches $98(1) \mathrm{mJ} / \mathrm{mol} \mathrm{K}^{2} \quad\left(\gamma=61 \mathrm{~mJ} / \mathrm{mol} \mathrm{K}^{2}\right.$ for UCoSn [14]), which reflects the increase of the density of states at the Fermi level $N\left(E_{\mathrm{F}}\right)$. The reason can be further localization of the electronic levels (but one has to keep in mind that the experimental $\gamma$-value reflects $N\left(E_{\mathrm{F}}\right)$ in the magnetically split state), the additional contribution of deuterium atoms to the total density of states, or the shift of the peaks of the electronic density.

In order to estimate the phonon contribution we have chosen the Debye model, which worked well in this case. The best fit was obtained for the model described by 9 branches (apparently the contribution of the metal atoms), with the Debye temperature of 217.5 K. The suggested model described the specific heat curve in the whole temperature range, while the extra specific heat in the proximity of the magnetic phase transition was evaluated to estimate the magnetic entropy as

$$
S_{\text {mag }}(T)=\int_{0}^{\mathrm{T}} \frac{C_{\text {mag }}\left(T^{\prime}\right)}{T^{\prime}} \mathrm{d} T^{\prime} .
$$

Such procedure yields a small magnetic entropy of $1.5 \mathrm{~J} \mathrm{~mol} / \mathrm{K}(0.26 * \mathrm{R} \ln 2)$, showing that the system is still far from the localization limit.

\section{Mössbauer spectroscopy}

Mössbauer spectra for $\mathrm{UCoSnD}_{0.6}$ were collected at room temperature and at $T=4.2 \mathrm{~K}$, i.e. in the paramagnetic region and in the magnetically ordered state.

The room temperature Mössbauer spectrum (Fig. 5) exhibits a broad peak, which could not be described by a single line. Therefore, a quadrupole splitting had to be considered in agreement with the 
Table 3 Estimated hyperfine parameters (isomer shift $I S$, quadrupole splitting $Q S$, hyperfine magnetic field $B_{\mathrm{hf}}$, asymmetry parameter of electric field gradient (EFG) $\eta, \theta$ and $\varphi$ polar and azimuthal angles specifying the direction of $B_{\mathrm{hf}}$ with respect to the main axes of the EFG tensor, and line-width $\Gamma$ ) from the ${ }^{119} \mathrm{Sn}$ Mössbauer spectra of $\mathrm{UCoSn}$ and $\mathrm{UCoSnD}_{0.6}$ obtained at different temperatures $T$.

\begin{tabular}{|c|c|c|c|c|c|c|c|c|}
\hline & $T(\mathrm{~K})$ & $I S(\mathrm{~mm} / \mathrm{s})$ & $Q S(\mathrm{~mm} / \mathrm{s})$ & $B_{\mathrm{hf}}(\mathrm{T})$ & $\eta$ & $\theta(\operatorname{deg})$ & $\varphi(\operatorname{deg})$ & $\Gamma(\mathrm{mm} / \mathrm{s})$ \\
\hline $\mathrm{UCoSn}^{\mathrm{a}}$ & 80 & 1.75 & 0.56 & - & - & - & - & 0.93 \\
\hline $\mathrm{UCoSnD}_{0.6^{\mathrm{b}}}$ & 300 & $1.78(1)$ & $0.64(1)$ & - & - & - & - & $0.96(2)$ \\
\hline $\mathrm{UCoSn}^{\mathrm{a}}$ & 4.2 & 1.765 & -0.55 & 8.40 & 0.22 & 90 & 90 & 0.76 \\
\hline $\mathrm{UCoSnD}_{0.6}{ }^{\mathrm{b}}$ & 4.2 & $1.89(4)$ & $-0.93(3)$ & $8.56(1)$ & 0.36 & 90 & 0 & $1.08(1)$ \\
\hline
\end{tabular}

$Q S$ was recalculated considering that for the $E_{\gamma}=23.875 \mathrm{keV}$ transition of ${ }^{119 \mathrm{~m}} \mathrm{Sn}, 1 \mathrm{~mm} / \mathrm{s}$ corresponds to 19.253(6) $\mathrm{MHz}$

${ }^{a}$ Data from [15]. The isomer shift $I S$ is relative to the $\mathrm{Ba}^{119 m} \mathrm{SnO}_{3}$ source.

${ }^{\mathrm{b}}$ Present work. The isomer shift $I S$ is relative to the $\mathrm{Ca}^{119 \mathrm{~m}} \mathrm{SnO}_{3}$ source.

low local symmetry of the Sn atoms. The hyperfine parameters extracted from the fit, describing two Lorentzians with equal line widths and relative areas, are given in Table 3. The lines are unresolved since the corresponding widths are larger than the quadrupole splitting. The hyperfine parameters of $\mathrm{UCoSnD}_{0.6}$ are close to those obtained for $\mathrm{UCoSn}$.

The Mössbauer spectrum of $\mathrm{UCoSnD}_{0.6}$ obtained at $T=4.2 \mathrm{~K}$ (Fig. 5) shows that, besides the main phase with a well-defined magnetic hyperfine field, there is a contribution from an impurity phase in the middle of the spectrum. Therefore it was necessary to reproduce the shape of the impurity component prior to the determination of the lines of the main phase, split by a magnetic hyperfine field. The hyperfine interaction parameters for $\mathrm{UCoSnD}_{0.6}$, obtained after this procedure, are presented in Table 3. In the magnetically ordered state, a non-zero magnetic hyperfine field is found on the Sn site for both UCoSn and the deuteride. The deuteration does not have significant impact on the value of the magnetic hyperfine field $B_{\mathrm{hf}}$, while the values of the quadrupole splitting and isomer shift (IS) are changed. The increase of $I S$ between room temperature and $T=$ $4.2 \mathrm{~K}$, deduced from the present data, was expected due to a second order Doppler effect. The $I S$ value is significantly lower compared to $\beta-\mathrm{Sn}(2.78(5) \mathrm{mm} / \mathrm{s})$, which corresponds to a lower $s$-state density at the $\mathrm{Sn}$ nuclei in the compound. This could be understood as due to the involvement of the $\mathrm{Sn}$ states in the hybridization. Then we can speculate that the increase of IS from UCoSn to $\mathrm{UCoSnD}_{0.6}$ is due to a reduced hybridization of the $\mathrm{Sn}$ states in the deuteride. The increase of the line-width for the deuteride compared to the initial compound is more pronounced at low temperature than for the samples measured at room temperature and might point to a higher degree of atomic disorder in the deuteride, or to a certain homogeneity region. Some changes were observed for the parameters of the electric field gradient (EFG). The direction of the main $z$-axis of the EFG does not change, but the $x$ - and $y$-axes are interchanged. For $\mathrm{UCoSnD}_{0.6}$ a slight improvement of the goodness of fit parameter was registered for the values $\theta=73^{\circ}$ and $\varphi=23^{\circ}$ of the polar and azimuthal angles defining the direction of $B_{h f}$ in the coordinate system of the EFG. This improvement is, however, not significant and therefore there is not enough evidence to suggest a deviation of the direction of the main axes of the EFG, which might be attributed to the presence of deuterium atoms in the crystal lattice. In fact, as they are not nearest neighbors of $\mathrm{Sn}$ atoms, their presence should not have a strong effect on the EFG at the Sn nuclei.

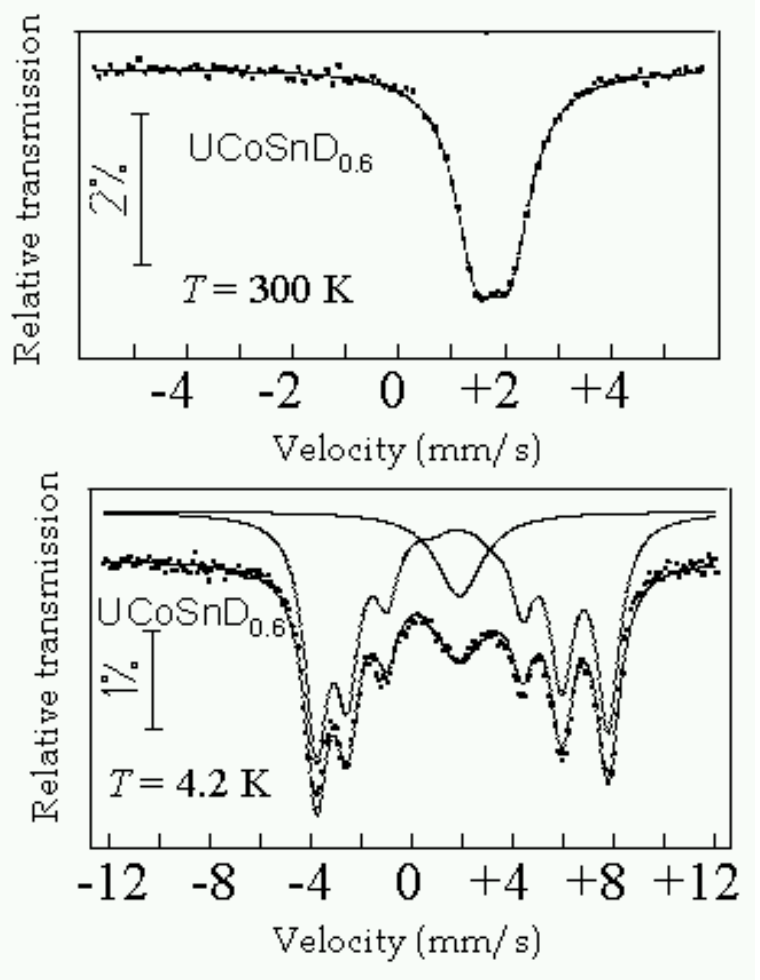

Fig. $5{ }^{119} \mathrm{Sn}$ Mössbauer spectra of $\mathrm{UCoSnD}_{0.6}$ taken at room temperature (upper panel) and at $T=4.2 \mathrm{~K}$ (lower panel). The calculated fit is shown by a solid line. 


\section{Conclusions}

The combined investigation of the crystal structure and physical properties of $\mathrm{UCoSnD}_{0.6}$ explained the strong effect of hydrogenation/deuteration on the magnetism of UCoSn. $\mathrm{UCoSnD}_{0.6}$ is a ferromagnet with an extremely high (for UTX ternary compounds, where $T$ - transition metal, $X-p$-element) Curie temperature, exceeding $100 \mathrm{~K}$. Based on the obtained results we may conclude that the mechanism responsible for the enhancement of magnetism in $\mathrm{UCoSn}$ is the decrease of the degree of $f-d$ hybridization, leading to a certain step in the localization of $5 f$ electronic states. The following facts support this idea: (i) the proximity of deuterium atoms to both $\mathrm{U}$ and Co must result in the formation of bonds, therefore withdrawing electronic density from the hybridized band; (ii) the increase of the density of states at the Fermi level can be a consequence of the band narrowing; (iii) the increase of the $s$-density of states on the Sn nucleus means again withdrawal of Sn electronic states from the hybridized band. This tendency was already observed for other uranium intermetallic compounds upon hydrogenation. For example, it was argued that the considerable increase of the ordering temperature of $\mathrm{UNiAl}$ after hydrogenation is due to a reduction of the hybridization effect [16]. Moreover, further studies of the $\mathrm{U}\left(\mathrm{Fe}_{1-x} \mathrm{Ni}_{x}\right) \mathrm{AlH}_{y}$ system $[17,18]$ clearly illustrated that hydrogenation strengthens the localization of uranium $5 f$ electrons. In the latter case hydrogenation induces magnetic order in the non-magnetic $\mathrm{U}\left(\mathrm{Fe}_{0.15} \mathrm{Ni}_{0.85}\right) \mathrm{Al}$ compound, and leads to a large increase of the ferromagnetic ordering temperature and the value of the uranium magnetic moment for the $\mathrm{U}\left(\mathrm{Fe}_{0.3} \mathrm{Ni}_{0.7}\right) \mathrm{Al}$ compound. However another issue still remains open - what is the reason for the different impact of hydrogenation on the magnetism of UCoSn and URuSn. It will be the subject of our future investigation.

\section{Acknowledgements}

This work was part of the research plan MSM 0021620834 financed by the Ministry of Education of the Czech Republic. It was also supported by the Grant Agency of the Czech Republic under grant No. 202/07/418 and by the action COST P16 under the project OC 146, financed by the Ministry of Education of the Czech Republic.

\section{References}

[1] A.E. Dwight, in: B.C. Giessen (Ed.), Developments in the Structural Chemistry of Alloy Phases, Plenum, New York, 1969, p. 181.

[2] A.V. Andreev, M.I. Bartashevich, Phys. Met. Metallogr. 62 (1986) 50.

[3] V. Sechovsky, L. Havela, G. Hilscher, N. Pillmayr, A.V. Andreev, P.A. Veenhuizen, F.R. de Boer, J. Appl. Phys. 63 (1988) 3070.

[4] V.H. Tran, R. Troc, J. Magn. Magn. Mater. 102 (1991) 74.

[5] V.H. Tran, R. Troc, D. Badurski, J. Alloys Compd. 219 (1995) 285.

[6] K. Miliyanchuk, L. Havela, A.V. Kolomiets, A.V. Andreev, J. Alloys Compd. 404-406 (2005) 165.

[7] J. Rodriguez-Carvajal, Physica B 192 (1993) 55.

[8] J.C. Waerenborgh, M.O. Figueiredo, J.M.P. Cabral, L.C.J. Pereira, J. Solid State Chem. 111(2) (1994) 300.

[9] K. Ruebenbauer, T. Birchall, Hyperfine Interact. 7 (1979) 125.

[10] R. Pöttgen, J.H. Albering, D. Kaczorowski, W. Jeitschko, J. Alloys Compd. 196 (1993) 111.

[11] T. Yamamoto, Y. Ishii, H. Kayano, J. Alloys Compd. 269 (1998) 162.

[12] D.P. Shoemaker, C. Brink Shoemaker, J. LessCommon Met. 68 (1979) 43.

[13] A.C. Switendick, Z. Phys. Chem., N.F. 117 (1979) 89.

[14] V. Sechovsky, L. Havela, L. Neuzil, A.V. Andreev, G. Hilscher, C.J. Schmitzer, J. Less-Common Met. 121 (1986) 169.

[15] R. Kruk, R. Kmiec, K. Latka, K. Tomala, R. Troc, V.H. Tran, Phys. Rev. B 55 (1997) 5851.

[16] H.N. Bordallo, H. Nakotte, A.V. Kolomiets, A. Christianson, L. Havela, A.J. Schultz, H. Drulis, W. Iwasieczko, Physica B 276-278 (2000) 706.

[17] P. Raj, A. Sathyamoorthy, K. Shashikala, N. Harish Kumar, C.R. Venkateswara Rao, S.K. Malik, J. Alloys Compd. 296 (2000) 20.

[18] P. Raj, K. Shashikala, A. Sathyamoorthy, N. Harish Kumar, C.R. Venkateswara Rao, S.K. Malik, Physica B 312-313 (2002) 88.

Proceeding of the X International Conference on Crystal Chemistry of Intermetallic Compounds, Lviv, September 17-20, 2007. 